\title{
Repensando el concepto de identidad política en la Argentina posneoliberal
}

\author{
Mauricio Schuttenberg*
}

\author{
Nivel: Comunicado \\ Recibido: 14 de mayo de 2012 \\ Aprobado: 20 de junio de 2012
}

\begin{abstract}
Resumen:
El artículo propone la reflexión en torno al concepto de identidad política aplicado a una investigación sobre las organizaciones populares en la Argentina contemporánea. Se intenta mostrar la centralidad del concepto de identidad en tanto permite entender las motivaciones y reconfiguraciones políticas que algunos movimientos tuvieron en el período posneoliberal. Asimismo, se presenta el proceso de operacionalización empírica de los conceptos y una aplicación en relación al análisis de la política reciente.

Palabras clave: Nacional, Popular, Identidad, Kirchnerismo
\end{abstract}

\begin{abstract}
Abstrac:
The article proposes the reflection on the concept of political identity applied to research on popular in contemporary Argentina organizations. It tries to show the centrality of the concept of identity as it allows to understand the motivations and political reconfigurations that some movements took in the period posneoliberal. Also, is the process of empirical operationalization of concepts and a proposal for implementation in relation to the recent policy analysis.
\end{abstract}

Keywords: National, Popular, Identity, Kirchnerismo

* Doctor en Ciencias Sociales por la Facultad Latinoamericana de Ciencias Sociales (FLACSO), Magíster en Ciencia Política por la Universidad Nacional de La Plata. Profesor Adjunto de Problemas de Historia Argentina en la Universidad Nacional Arturo Jauretche (UNAJ) y Jefe de Trabajos Prácticos en Historia de las Ideas y los procesos políticos en la Facultad de Periodismo y Comunicación Social de la Universidad Nacional de La Plata. Integrante de los centros CPS e IdIHCS en la UNLP. mauricioschuttenberg@gmail.com. 


\section{Introducción}

En los últimos años el concepto de identidad aparece como referencia de una gran cantidad de estudios sociales de temáticas diferentes. No sólo los diversos campos donde se aplica el concepto, sino más bien la polisemia con la cual se lo utiliza se presenta como un problema a tener en cuenta para el análisis social.

Este artículo propone dos objetivos centrales. En primer lugar, presentar un marco conceptual utilizado para el análisis de las identidades políticas de las organizaciones "nacional populares"1 en su complejidad, que permitió la reflexión en torno a los actos de identificación, las decisiones, los imaginarios sociales y las reconfiguraciones identitarias de tales organizaciones. ${ }^{2}$ La relevancia de estas cuestiones se sustenta en que es la identidad la que posibilita a los actores significar las situaciones y escoger en consecuencia ciertas alternativas de acción política. En segundo término, se presenta una operacionalización de categorías teóricas con las cuales se abordó la dinámica política de dichas organizaciones durante el período 2003-2009, y algunos resultados generales de la investigación.

En 2003 con la asunción del presidente Néstor Kirchner y la generación de un entramado político transversal que incluyó entre sus filas a organizaciones "piqueteras"3", marcó importantes

1 El campo "nacional popular" se constituye a partir de un conjunto de experiencias, imaginarios, figuras, mitos, símbolos y relatos ligados al peronismo, al nacionalismo revolucionario, al campo de las izquierdas y a miradas revisionistas de la historia, a los que las organizaciones apelarán de distinta forma configurando así sus identidades. Este "telón de fondo" es resignificado y atravesado por las identidades de las organizaciones que tomarán esos elementos articulando sus cadenas de significación.

2 El presente trabajo retoma las herramientas teóricas de la tesis de doctorado en Ciencias Sociales de FLACSO "La reconfiguración de las identidades "nacional populares" durante el kirchnerismo 2003-2009. Estudio de tres organizaciones: Libres del Sur, Movimiento Evita y Movimiento de Unidad Popular", dirigida por el Dr. Aníbal Viguera y codirigida por el Dr. Martín Retamozo, a quienes se agradece las observaciones y comentarios.

3 Las organizaciones de Trabajadores Desocupados fueron denominadas también 
mutaciones en las acciones de protesta y en los alineamientos y configuraciones identitarias de varias organizaciones en torno a distintas variantes de una identidad "nacional-popular" a partir de la convergencia y resignificación de diversas tradiciones políticas. El artículo apunta a pensar las identidades "nacional populares" no como una identidad "esencial" sino como una construcción histórica en donde las organizaciones ponen en juego procesos de construcción identitaria que incluyen elementos sedimentados a partir de sus experiencias políticas previas y elementos que se activarán en cada coyuntura reconfigurando sus marcos de interpretación.

El cambio del escenario político a partir de 2003 generó en efecto una importante modificación en el modo de vinculación entre Estado y organizaciones de desocupados. La estrategia que desarrolló el gobierno de Kirchner fue incorporar a su proyecto político a algunas de esas organizaciones, lo que a su vez potenció, reactualizó y resignificó la posibilidad de que ellas se articulen en torno a una identidad "nacional-popular". Ahora bien, la inserción de algunos grupos en el espacio kirchnerista tendió a explicarse en un principio, en numerosos trabajos del período (Campione y Rajland, 2006; Borón, 2007; Battistini, 2007, Svampa, 2006), en términos de cooptación, concepto que desde mi perspectiva resulta insuficiente para dar cuenta de los posicionamientos de un número importante de organizaciones en tanto subyace en él un argumento negador de la producción política de estos actores.

Si bien esta idea es el denominador común del proceso político abierto en 2003, aparece formulada haciendo hincapié en

como "piqueteros", a partir de la modalidad de protesta con el corte de ruta en su surgimiento a mediados de los años 90. Estos movimientos articularon demandas de inclusión, trabajo y transformación social e impugnaban fuertemente el modelo económico neoliberal vigente en la Argentina de esos años. No obstante, el movimiento piquetero es un conjunto heterogéneo de organizaciones con diversas ideologías y formatos organizativos. 
distintas causas. Se la caracterizará como estrategia estatal de contención de la protesta, como reemplazo al nivel colectivo de la matriz clientelar, como manifestación de la debilidad de los sectores y organizaciones populares y como capacidad de "volver al orden" del Partido Justicialista ${ }^{4}$.

Desde esta perspectiva teórica, la idea de cooptación explica entonces el posicionamiento y el reordenamiento político posterior a 2003. No obstante, en los últimos años salieron a la luz algunos trabajos que comenzaron a cuestionar las hipótesis de cooptación y empezaron a problematizar la dinámica de los movimientos sociales en la presidencia de Kirchner. Esta relación comienza a ser interpretada como una decisión conciente de las organizaciones ante el desafío de reposicionarse frente a un contexto de reflujo de la movilización y, fundamentalmente, a redefinir sus estrategias políticas frente a un gobierno que construyó rápidamente su legitimidad de ejercicio apelando a la oposición al modelo neoliberal a través de un imaginario productivista y distributivo que recuperaba buena parte de las demandas que habían permitido la articulación de la protesta (Pérez, 2008; Massetti, 2009 y Gómez, 2009).

La mayoría de los trabajos que abordan la etapa posterior a 2003 dan cuenta del paso del ciclo de protesta a una mutación de la misma a partir de la asunción de Kirchner, poniendo el acento en cómo desde el Estado se condicionó la acción política de las organizaciones. No se ha indagado en la misma medida lo que ocurrió "abajo", es decir, cómo se vivenció en las organizaciones la etapa que se abrió en 2003 y cómo algunas de ellas construyeron nuevos posicionamientos y rearticularon en el camino nuevos y viejos componentes identitarios.

4 El Partido Justicialista durante los años 90 implantó una serie de reformas neoliberales bajo la presidencia de Carlos Menem. Ese mismo partido impulsará una serie de reformas que darán lugar a un escenario posneoliberal. 
En este marco, el objetivo que el trabajo propone es recuperar la historia de esas organizaciones y comprender que la dinámica política que comienza en 2003 no es necesariamente para ellas una ruptura, cooptación y abandono de prédicas revolucionarias, sino que se trata de un proceso de construcción y reconstrucción de las identidades "nacional populares", en donde se pone en juego la historicidad sedimentada de las organizaciones en distintos contextos. Esto posibilita desarrollar una lectura de largo plazo de las mutaciones de las identidades de las organizaciones para captar los elementos de continuidad y los antecedentes precursores que se corresponden con desarrollos y posicionamientos ulteriores.

Siguiendo este razonamiento podríamos preguntarnos: ¿Qué espacios políticos se abrieron a partir de 2003 para algunas organizaciones populares? ¿En qué se sustentan las nuevas interpretaciones que las organizaciones realizan de las nuevas relaciones con el Estado? ¿Cómo se resignifica el pasado y las tradiciones políticas en las lecturas del presente? ¿Desde qué lugares se reivindica la identidad peronista? ¿Cómo son las instancias de producción de identidad? Para contestar estas preguntas era ne-

5 Dentro del recorte que se abordó en la tesis se tomaron: El movimiento Evita de 2005 se formó a partir de la confluencia de organizaciones y movimientos entre las cuales se encontraban el Movimiento de Trabajadores Desocupados Evita, el MTD Resistir y Vencer, las 4 P (Pan y Poder para el Pueblo), una escisión de MPRQ (Movimiento Patriótico Revolucionario Quebracho), el MP 20 (Movimiento Patriótico 20 de Diciembre), la organización estudiantil 20 de Febrero (fecha que hace alusión a la lucha de resistencia a la implementación de la ley de Educación durante febrero de 1996), Peronismo que Resiste, y sectores del Partido Justicialista. El movimiento Libres del Sur se lanzó oficialmente el 27 de abril de 2006 en un acto en el Centro Costa Salguero de la Ciudad de Buenos Aires. El movimiento se conformó a partir de la fusión del Movimiento Barrios de Pie (brazo territorial de la Corriente Patria Libre), la Corriente Patria Libre, el Partido Comunista Congreso Extraordinario, la Agrupación Martín Fierro, el Frente Barrial 19 de Diciembre y la Agrupación Envar el Kadri.

El Movimiento de Unidad Popular (MUP) surge en 1999 a partir de una organización de vertiente anarquista llamada AUCA (rebelde en mapuche). Este movimiento de raíz libertaria se inició a fines de los años 90 en barrios de La Plata y fue creciendo hacia otras provincias y el conurbano bonaerense. El MUP nacía como el "movimiento de masas" de dicha línea política. 
cesario construir un "lente teórico" sensible a los procesos de reconstrucción identitaria de esas organizaciones que, surgidas en la lucha contra el neoliberalismo, construyen sus vínculos con el gobierno de Kirchner.

\section{El problema de la identidad en los movimientos sociales}

La necesidad de analizar los movimientos sociales para las ciencias sociales se dio desde el momento en que muchas de las certezas acerca de las identidades colectivas homogéneas ${ }^{6}$ se fueron desmoronando (Schuster, 2005). En "las nuevas sociedades" reflexivas (Beck, 2002 y 2004), cambiantes, el conflicto parece diseminarse por todo el espacio social y no será ya, sólo, el movimiento obrero el sujeto político que encarne el conflicto societal.

En ese contexto cobraron forma dos vertientes fundamentales, una en Estados Unidos -conocida en principio como "teoría de la movilización de recursos"- y la otra en Europa -o teoría de los nuevos movimientos sociales propiamente dicha-, cada una proponiendo contribuciones particulares al análisis de los movimientos ${ }^{7}$. No obstante, es necesario aclarar que las perspectivas europeas y norteamericanas no representan "escuelas" teóricas unitarias, sino que defienden diferentes interpretaciones en sus explicaciones sobre el surgimiento y desarrollo de los movimientos sociales desde los setenta (Klandermans y Tarrow, 1988, cita-

6 La etapa previa a los años '60 y '70, se caracterizaba por la tendencia a la formación de grandes actores colectivos fundados e identificados con el mundo del trabajo. En el plano económico social, las ideologías intervencionistas y dirigistas, con algunos rasgos distintivos derivados de sus historias nacionales, fueron un elemento presente en la mayoría de las elites gubernamentales. El cierre de esta etapa mundial tuvo como principales procesos: en el Oeste, la crisis del Estado de Bienestar y la disminución de la importancia política y económica de la clase obrera industrial; en el Este, los colapsos de los socialismos reales; en el Sur las transiciones a la democracia (Sidicaro, 2003).

7 La bibliografía sobre estas teorías es amplia y variada. Una síntesis bibliográfica de temas y autores puede encontrarse en Jenkins (1994). 
do en Rubio García, 2004). Si en las propuestas norteamericanas era el carácter estratégico de la acción colectiva lo que proporcionaba el principal nexo de unión, formular los nuevos movimientos sociales como la reacción a los cambios estructurales de las sociedades avanzadas será el elemento unificador de los teóricos europeos. Las diferencias entre las dos escuelas de pensamiento obedecían a las tradiciones intelectuales dominantes en cada cultura: individualista en Estados Unidos y estructuralista en Europa (Tarrow, 1997 y 1999).

En la vertiente norteamericana, las investigaciones sobre los movimientos sociales se centraron en sus formas de acción política y sus posicionamientos estratégicos que fueron analizados desde el concepto de acción colectiva. Esta noción apuntaba a dar cuenta de las motivaciones por las cuales se producía efectivamente la movilización de colectivos. En este marco, en un primer momento la cuestión de la identidad de los movimientos sociales quedó relegada en la búsqueda de elementos "externos" que pudieran explicar la movilización y la acción política. La acción entonces se explicó a partir de las oportunidades políticas y la aparición coyuntural en el sistema político de elementos que promueven la participación.

No obstante, luego aparecieron conceptos que apuntaban a explicar la movilización desde la indagación de las motivaciones "internas" de los movimientos. Así surgieron los conceptos de estructuras de la movilización y los marcos estratégicos que miraban hacia el interior de las organizaciones en la búsqueda de explicaciones de la acción.

Allí encontraremos entonces algunos elementos que irán desarrollándose ligados a la cuestión de las identidades pero atravesados por una constante de la escuela norteamericana que es la de estrategia ${ }^{8}$. Todas las construcciones simbólicas, "las ban-

8 Según esta perspectiva, la sociedad es un mercado de recursos económicos, sociales e ideológicos y tanto los actores inconformes como sus adversarios 
deras", de las organizaciones fueron pensadas en términos de construcciones con fines específicos, o elementos a los que, mediante una operación estratégica, los líderes tomaban con un fin determinado.

Los conceptos centrales de la teoría del enfoque político ${ }^{9}$ son los de estructura de oportunidades políticas, las estructuras de movilización y la construcción de procesos enmarcadores. El primero de los conceptos mencionados, es decir, el de la estructura de oportunidades políticas, es definido como "dimensiones congruentes -aunque no necesariamente formales o permanentes- del entorno político que ofrecen incentivos para que la gente participe en acciones colectivas al afectar a sus expectativas de éxito o fracaso" (Tarrow, 1997: 155). El concepto de estructura de las oportunidades políticas ayuda a comprender por qué los movimientos adquieren en ocasiones una sorprendente, aunque transitoria, capacidad de presión contra las elites o autoridades y luego la pierden rápidamente a pesar de todos sus esfuerzos.

Sin embargo, es importante resaltar que para este autor la visión de la oportunidad política no es algo estático en el sentido que habría que esperar el momento de golpear, sino más bien que la oportunidad política es también un proceso abierto en el cual los grupos la van generando. Es así que la ampliación de las oportunidades para un determinado grupo puede ir de la mano de la creación de oportunidades para los oponentes a ese grupo o para

desarrollan estrategias racionales para obtener la satisfacción de sus demandas o proteger sus intereses. De esta manera, el proceso central es el de la movilización de recursos que se conforma, por un lado, alrededor de individuos insatisfechos con un orden, que acumulan fuerza y desarrollan estrategias para incrementarla, y, por otro, por actores que defienden el orden y manejan el control social (Tarrés, 1992: 744).

9 La Teoría de los procesos políticos es, de alguna manera, subsidiaria de la Teoría de movilización de recursos que tiene como rasgo central el componente instrumental de la racionalidad estratégica como criterio de maximización de utilidades, con el cual se evaluará la relación costo-beneficio de implicarse individualmente en la acción colectiva. El enfoque político incorpora sus principales supuestos y profundiza el análisis en torno de la organización que se moviliza y el contexto en el que se produce (Armelino, 2007). 
las elites mismas. En esta visión las oportunidades políticas aparecen escasamente problematizadas en relación a la identidad de los movimientos. ¿De qué depende que los individuos interpreten como una oportunidad un determinado proceso político? Si pensamos a las oportunidades no como una situación "objetiva" sino más bien como una construcción que los grupos realizan, veremos la importancia que cobra el concepto de identidad y cómo a partir de allí las oportunidades políticas serán concebidas en un marco más amplio de significaciones (Mc Adam, 1999: 27). En este enfoque, entonces, la cuestión identitaria no sería un producto del recorrido histórico de las mismas, o mejor dicho producto de su historicidad y de sus luchas pasadas, sino más bien una construcción realizada en pos de un objetivo político particular.

Por otro lado, el problema de la identidad fue abordado como el eje central por la denominada teoría de los movimientos sociales o vertiente "europea". Se concibe como una contrapropuesta para comprender las luchas y reivindicaciones sociales a partir del rescate del actor y del sujeto. La perspectiva de los nuevos movimientos sociales que se origina en Europa trata de poner en evidencia las dimensiones culturales y sociales de las prácticas colectivas. Para esta línea los actores sociales, por medio de sus prácticas colectivas, reinterpretan normas y valores, creando nuevos significados para los estrechos límites de la acción política. Esta línea de investigaciones compartía un desencanto con el marxismo estructural que privilegiaba el análisis de las contradicciones de las clases definidas desde la economía. También les interesaba comprender las movilizaciones que se desarrollaban en sus países a fines de los años sesenta, las cuales aparecieron como nuevas y distintas de las anteriores analizadas por la izquierda política.

Es necesario remarcar que esta mirada, aunque parte del concepto de identidad, surgió en un contexto muy diferente al de las organizaciones populares de la Argentina reciente. Estos estudios analizaron a actores movilizados que provenían de las clases 
medias y las demandas se relacionaban con la democratización de la vida social y la redefinición de la esfera privada, dejando de lado temas como la transformación económica o el juego por el control del poder estatal ${ }^{10}$. El eje de estos autores dedicados a los "nuevos movimientos sociales" se basó en una crítica a las teorías que buscaban un principio de unidad, un lugar central de la sociedad para explicar la acción social. De esta forma, la categoría de identidad será crucial para dar cuenta de la definición que el actor hace de sí mismo y la definición que hará de sus adversarios.

La perspectiva de los movimientos sociales buscó comprender la lógica de la acción colectiva en las llamadas sociedades europeas posindustriales. La contribución de esta perspectiva fue que rescató a sectores sociales que habían sido borrados por la aplicación de modelos estructuralistas, o por la implacable lógica de la teoría de las clases sociales. De esa forma se descartaban las explicaciones que daban por supuesto el comportamiento de ciertos actores (Tarrés, 1992: 736).

Melucci (1994: 150) criticó a la teoría americana porque al poner el énfasis en la movilización de recursos reducía el análisis de los movimientos al puro terreno de la política, y, en concreto, a la confrontación con el sistema político y la intervención en las decisiones políticas, subestimando la creación de códigos culturales sobre los cuales se asientan las acciones públicas. Por otra parte, este enfoque descuidaba el análisis de las causas últimas que provocan la movilización bajo el argumento de que los mo-

10 Offe (1992) marcaba como diferencias de los nuevos movimientos sociales con los viejos que sus actores, en su autoidentificación, no se refieren a códigos políticos establecidos ni a códigos socioeconómicos, sino a atributos del género humano en su conjunto, y ello les distingue de los anteriores movimientos. Afirma que el enfoque de los Nuevos Movimientos Sociales es un modelo de política sin un proyecto construido teóricamente, y de allí proviene la naturaleza fragmentaria, ad hoc, pluralista y selectiva y su rechazo a "ideologías totalizadoras", lo que hace que se tienda a dejar de lado el debate ideológico (Klachko, 2008). 
tivos de las quejas son permanentes y sólo cambian los recursos disponibles (Pérez Ledesma, 1994).

El eje para Melucci (1994: 154) es que los fenómenos colectivos son resultado de múltiples procesos que favorecen o impiden la formación y el mantenimiento de las estructuras cognoscitivas y los sistemas de relaciones necesarios para la acción. De esta forma, el fenómeno colectivo es producto de procesos sociales diferenciados, de orientaciones de la acción, de elementos de estructura y motivación. Para este autor faltaba el análisis de los procesos a través de los cuales los individuos evalúan y reconocen lo que tienen en común y deciden actuar conjuntamente, "fruto del reconocimiento emocional" (Melucci, 1994: 155). Esta teoría pone el énfasis en el proceso de construcción de un sistema de acción colectiva donde los actores producen estructuras cognoscitivas comunes que se denominan identidad colectiva. La identidad colectiva puede cristalizar en formas organizacionales, sistemas de reglas y relaciones de liderazgo.

Este paradigma analizó cómo los grupos buscan, a través de sus acciones, obtener autonomía, reconocimiento y afianzar un proceso identitario en sociedades modernas que ganan en complejidad (Touraine, 1991; Melucci, 1994, 2002; Pizzorno 1994). Los conflictos surgidos desde los ochenta reflejan estos nuevos rasgos de la acción colectiva, donde se evidencia que sus temáticas son particulares, que sus actores son temporales, que dan un lugar central a la expresión simbólica y que no buscan principalmente metas materiales. De esta forma, la escuela europea tendió a destacar los objetivos sociales y culturales mostrando menos preocupación por entender la relación con la arena político-institucional. En tanto la bibliografía norteamericana se centró más en la relación entre los movimientos y la esfera política y menos en la dimensión social de los movimientos.

Estas discusiones entre estos dos paradigmas de la acción colectiva dieron lugar luego a la necesidad de articulación entre los mismos. Diversos autores destacarán la necesidad de producir 
puentes o acercamientos entre ambos con el fin de tener una visión más compleja de los movimientos, lo que, situándose sólo en una de las perspectivas, no sería posible (Munck, 1995: 17; Schuster, 2005: 49).

No obstante, a la hora de pensar un andamiaje teórico para el análisis de las organizaciones populares en la Argentina contemporánea, los conceptos desarrollados anteriormente de las teorías del enfoque político y de la movilización de recursos, resultan poco sensibles para el análisis de los procesos constituyentes y de las reconfiguraciones identitarias de las organizaciones populares por prestar escasa atención a la dimensión simbólica de las mismas, subsumiendo esa producción detrás de una concepción más estratégica de la política.

Asimismo, los enfoques identitarios como los de Melucci, Pizzorno y Touraine, basados en el concepto de la identidad colectiva de los nuevos movimientos sociales, necesitan una "adaptación" para el análisis de organizaciones que se caracterizan por la construcción de fuertes vínculos y tradiciones con formas de organización política que pueden ser vistas como una continuidad de reivindicaciones centradas en la defensa de los derechos de los trabajadores, y no en demandas particulares como sería la característica de los movimientos sociales (Gurrera, 2005: 18).

Por lo tanto, estas miradas requieren ser complementadas por otras perspectivas a la hora de pensar la identidad de las organizaciones de trabajadores desocupados y las organizaciones populares que de ellas se desprenden. En estos casos "lo político" aparece como central, tienen además proyectos ideológicos y no son fragmentarios. Asimismo, estas identidades no sólo no rechazan las totalizaciones sino que las reconstruyen. Por ende, se hace necesaria la conceptualización de una matriz analítica capaz de dar cuenta de la especificidad de las reconfiguraciones identitarias de las organizaciones populares ocurridas en los últimos años en la Argentina. 
En este sentido, la idea de identidad colectiva ${ }^{11}$ como instancia de creación horizontal y de carácter más coyuntural que tienen los estudios europeos parece insuficiente para la comprensión de las reconfiguraciones identitarias de las organizaciones políticas que este trabajo toma. La presencia de jerarquías, de lógicas de organización ligadas a lo partidario, la existencia de identidades políticas con demandas de carácter estructural-económico, hacen necesario pensar un concepto de identidad política en organizaciones que dé cuenta de la historicidad de estas, de sus demandas y los procesos dinámicos de construcción y reconfiguración.

\section{El aporte del concepto de identidad política para el estudio de organizaciones populares}

Investigar la dinámica política de las organizaciones populares durante la etapa posneoliberal implica pensar los procesos de reconfiguración de las identidades políticas y exige "adaptar" ciertos conceptos a un campo de aplicación diferente al de movimiento social. Analizar los procesos constituyentes (Zemelman, 1997) y no reducir el análisis de las organizaciones políticas simplemente a la condición de productos cristalizados en las diferentes etapas históricas, nos permitirá dar cuenta de los dinamismos a fin de evitar una mirada estática de las identidades.

Cabe señalar que en los estudios sobre la acción colectiva popular en la Argentina reciente ha prevalecido el estudio de las

11 Otros estudios operacionalizaron de distinta forma el concepto de identidad. Así en algunos casos, el análisis de trayectorias iba detrás de la identidad individual. Esta concepción tiene que ver con la idea que tenemos acerca de quiénes somos y quiénes son los otros, es decir, con la representación que tenemos de nosotros mismos en relación con los demás. La identidad puede definirse en esta mirada como un proceso subjetivo (y frecuentemente auto-reflexivo) por el que los sujetos definen su diferencia de otros sujetos (y de su entorno social) mediante la auto-asignación de un repertorio de atributos culturales frecuentemente valorizados y relativamente estables en el tiempo (Giménez, 1997 y 2004, y Dubar, 1991). Cabe destacar en esta línea de investigación los conocidos trabajos de Bauman (2002) y su concepción de la modernidad "líquida". 
identidades en los denominados movimientos sociales, con un énfasis en la identidad colectiva en su dimensión de identidad social $^{12}$, en tanto representantes de demandas de desocupados; el análisis de la identidad política de estas organizaciones ha recibido una atención menor. En este sentido un primer paso a dar es especificar y diferenciar el concepto de organizaciones populares respecto al de movimientos sociales, para luego explorar las herramientas analíticas que nos permitan estudiar las identidades en su dimensión política. La definición de un movimiento social se basa en el proceso de constitución de una identidad colectiva, fuera del ámbito de la política institucional, por el cual se dota de sentido a la acción individual y colectiva (Revilla Blanco, 1994: 11). Schuster (2005: 48) define a un movimiento social a partir de una serie de características tales como tener una identidad común, que dicha identidad tenga continuidad en el tiempo, que exista una expansión en el espacio del sistema identitario de acciones y que la línea de identidad de las acciones pueda reconstruirse a través de sucesivas emergencias y ocultamientos.

El concepto de movimiento social alude fundamentalmente a tres características que son: la identidad en común a través del tiempo, la construcción de demandas que exceden las reivindicaciones de clase y una estructuración no jerárquica. En este trabajo nos referiremos a organización en lugar de movimiento social puesto que si bien comparte un aspecto de la definición del mismo, es decir, la existencia de una sólida identidad colectiva, la

12 Esta perspectiva de las identidades sociales tuvo desarrollo en los estudios que, para el caso de la Argentina, trataron la "territorialización" de los vínculos y la acción colectiva de las clases populares (Svampa, 2000, 2004, 2005 y Merklen, 2005). El barrio aparece entonces como referencia de la identidad, en la medida que sus pobladores, al construirlo, generan lazos de pertenencia, que les permite distinguirse frente a otros colectivos sociales. También en él se construyen diferentes identidades colectivas, que expresan la fragmentación y las diferencias propias de la vida urbana contemporánea (Torres Carrillo, 2006: 8). Asimismo, otros trabajos se centraron en pensar las identidades sociales a partir de analizar la articulación de demandas de los movimientos en tanto instancias de representación de trabajadores desocupados. 
cuestión de la horizontalidad y el carácter "estructurado" de las mismas son elementos que las alejan de esa concepción.

La noción de organizaciones populares hace referencia a un espacio de conformación en el intersticio que dejaron los partidos políticos y los sindicatos (Masetti, 2009: 122), y en su origen se encuentran dos procesos: el desarrollo de un nuevo paradigma de acumulación política surgido a nivel local y las experiencias colectivas de resolución de necesidades a partir de las crisis económicas de los años 80 y 90 . El concepto de "organizaciones populares" se ubica en una dimensión analítica diferente a la de movimiento social en tanto hace alusión a un referente empírico concreto más que a lógicas de acción. A diferencia de los movimientos sociales, las organizaciones se caracterizan por: poseer un relativo grado de estructuración organizativa que retoma antiguas tradiciones e identidades políticas, articular demandas de carácter económico y posicionarse y trazar estrategias en el ámbito político institucional.

Partir entonces de la pregunta acerca de la reconfiguración de las identidades políticas conlleva a establecer algunas definiciones previas de lo que ello implica. Debido a que el término "identidad" parece semánticamente inseparable de la idea de "permanencia", es necesario pensar un concepto capaz de captar tanto "lo permanente" (sedimentado) de una identidad política como su continua recreación, es decir, sus dimensiones estáticas y dinámicas. Buscamos reconstruir las mutaciones y a su vez lo sedimentado que estructura una identidad política.

En este trabajo apuntamos a pensar el campo "nacional-popular", no como una identidad esencial sino como una construcción histórica en donde las diversas identidades ponen en juego procesos de construcción que incluyen elementos sedimentados a partir de sus experiencias políticas previas y elementos que se activarán al calor de la coyuntura y las alternativas del proceso político. 
Para analizar las formas de constitución y reconfiguración de las identidades políticas "nacional populares", se tomaron algunos conceptos claves de Laclau como: hegemonía, antagonismo, puntos nodales y significantes vacíos. La importancia del análisis del discurso parte del principio de comprender a lo social como un espacio discursivo. Desde esta perspectiva, el discurso no sería producido por un sujeto que fuera su agente, sino a la inversa, el sujeto social sería una realización del discurso (Biglieri, 2007: 37).

La identidad implica entonces un proceso dinámico de construcción de significados. En este punto recuperamos el concepto de configuración identitaria propuesto por De la Garza (1997, 2001). Entender la identidad como una configuración permite concebirla como un proceso móvil que articula elementos heterogéneos que tendrán distintos lugares en esa cadena significante en las distintas coyunturas.

Estos procesos constituyentes permiten pensar que la reconstrucción de las identidades y la atribución de sentidos por parte de las mismas, se establecen como elementos centrales para el análisis de los posicionamientos políticos. Los elementos constitutivos de la identidad se articulan en una dinámica siempre inacabada y abierta (Zemelman, 1995: 11). Esto implica la necesidad de analizar una dimensión autónoma de los aspectos estructurales que inciden en los mecanismos de identificación y en la conformación de configuraciones identitarias.

Pensar las identidades en estos términos abre la posibilidad de entender la conformación de nuevas configuraciones. No obstante, cada reordenamiento, cada incorporación, cada modificación, cada reconfiguración, genera reacomodamientos donde se pueden identificar continuidades y cambios (Barros, 2006b). Dentro de esos elementos que se reconfiguran tiene importancia el análisis de los puntos nodales donde se condensan los significados. En efecto, en la configuración identitaria no todos los significados tienen el mismo peso para la articulación, algunos códigos pueden adquirir primacía y opacar a otros que perma- 
necen subalternizados, pero que pueden emerger y conformarse en "articulantes" de la red de códigos (Retamozo, 2009a y 2009b). Esos puntos nodales constituyen de alguna forma significantes centrales que estructuran una cadena significante y estabilizan temporariamente una identidad (Laclau, 2006: 3).

Esto último es central, puesto que las identidades nunca logran una sutura "definitiva" y están siempre en redefinición. Las organizaciones transforman su propia identidad en la medida que actualizan ciertas posibilidades y desechan otras. Siendo que toda identidad es contingente y esencialmente relacional respecto de sus condiciones de existencia, ningún cambio de estas últimas puede dejar de afectar aquella. Por ello, la reformulación hegemónica cambia la identidad de todas las fuerzas sociales en presente (Laclau, 1990: 47).

A la hora de "bajar" este marco teórico al plano empírico, se planteó reconstruir los puntos nodales sobre los cuales las organizaciones bajo estudio construyeron su identidad y cómo estos fueron mutando en las diferentes etapas. La forma de operacionalizar este concepto se basó en la búsqueda de las concepciones que las organizaciones fueron desarrollando sobre el Estado, la democracia, sobre el sujeto histórico del cambio social, sobre cómo debía darse esa transformación social, sobre las interpretaciones de la historia, las alteridades y límites discursivos, sus lecturas respecto al peronismo y en los mitos fundantes de las diversas tradiciones ${ }^{13}$.

13 Para el análisis se tomaron documentos públicos y de circulación interna de las tres organizaciones. En tanto también se trabajó con las publicaciones que las organizaciones tuvieron en las distintas etapas: Revista Evita del Movimiento Evita, Revista En Marcha de Patria Libre y Revista Patria Grande de Libres del Sur, y con respecto al MUP se tomaron centralmente los documentos en los diversos períodos abordados. A su vez se realizaron entrevistas semiestructuradas a dirigentes de las tres organizaciones desde agosto de 2007 a 2009. Los cuestionarios apuntaban a recabar información sobre las distintas interpretaciones, posicionamientos que las organizaciones analizadas tuvieron desde su conformación hasta el presente y sobre las trayectorias y tradiciones de la militancia que las compone. 
Estos elementos identificados apuntaron a enfatizar en los núcleos donde se sedimentan los sentidos de estas identidades. La descripción profunda de estos puntos nodales nos permitió acceder a los elementos acumulados por las experiencias políticas previas a 2003 y cómo éstas tomarán nuevas significaciones en el contexto del gobierno de Néstor Kirchner.

En este plano se tomaron en cuenta las trayectorias en donde se destacaron tres afluentes temporales en la militancia que fueron configurando una identidad en cada una de las organizaciones. El primero es la experiencia militante de los años 70 en tanto antiguos cuadros del Partido Revolucionario de los Trabajadores (PRT) abrevarán a Libres del Sur, y militantes de Montoneros serán parte integrante del Movimiento Evita. Un segundo centrado en las experiencias políticas de los ‘ 80 y, por último, la impronta que trajo consigo la aparición, en la década de los '90, de los Movimientos de Trabajadores Desocupados. En este punto es importante destacar que a diferencia del Movimiento Evita y Libres del Sur que se nutren de los distintos afluentes temporales de militancia, el Movimiento de Unidad Popular tiene su origen fundante en la década de los noventa y se conformó a partir de militantes jóvenes que cuestionaban las representaciones existentes dentro del panorama político partidario de la época.

El análisis documental y de las trayectorias de la militancia nos permitió observar la conformación de las organizaciones y las tradiciones que reunirán cada una de ellas. De esta forma Libres del Sur se constituyó como "izquierda nacional revolucionaria" lo que implicaba compartir ciertos elementos simbólicos con el espacio "nacional popular" aunque guardaban algunas diferencias en torno a la caracterización del peronismo-pejotismo y a las posibilidades históricas del primero. El Movimiento Evita reagrupó los núcleos militantes ligados al peronismo revolucionario fragmentados en la dinámica de los años '90 y el Movimiento de Unidad Popular surgió como organización de jóvenes "anarquistas libertarios" con una fuerte prédica latinoamerica- 
nista y que se desvinculaban de la tradición anarquista argentina de fines del siglo XIX y principios del XX.

Lo central de la etapa de construcción identitaria de finales de los años 90 es la conformación, en los tres grupos de organizaciones, de una cadena de sentido que articulaba a los significantes Estado y Gobierno en un espacio de alteridad. Esa cadena consolidó un antagonismo en donde, en la visión de las organizaciones, el Partido Justicialista estaba dentro del "campo enemigo". Esos significantes históricamente estructurados se reactivarán y reconfigurarán en la etapa posterior a 2003 constituyendo un acercamiento en distintas etapas, para luego dar lugar a una articulación en constante tensión con esa otredad que les representaba el kirchnerismo.

Ahora bien, esas tradiciones conformadas se reconfiguraron en la coyuntura de 2003 que inauguró una etapa en donde las organizaciones participarán del gobierno de Néstor Kirchner. Esa relación se forjó a partir de una compleja articulación política que denominamos "puentes discursivos". Estos puentes expresan el pasaje de una determinada configuración identitaria a otra, explican la reconstrucción que cada identidad realiza de sí misma en cada coyuntura. Esta instancia de inclusión marcó la tensión compleja entre la ampliación y vaciamiento de los principales elementos significantes de las configuraciones identitarias en busca de la articulación con el espacio "kirchnerista" y la "custodia" de elementos significantes centrales que constituirán límites que, en determinadas coyunturas, marcarán la imposibilidad de esa articulación.

Los "puentes discursivos" marcaron las formas en las cuales se produjo la inserción de las organizaciones en el Gobierno. Estos puentes implicaron la producción de una compleja operación política de articulaciones y reconfiguraciones identitarias en donde se entretejieron las trayectorias previas, las identidades y las expectativas respecto a la participación en el nuevo espacio. Las organizaciones se vieron en la necesidad de redefinir su po- 
sicionamiento para afrontar lo que entendían era una etapa diferente de la historia ante la llegada de Kirchner al gobierno, de la mano de un discurso "reparador". La resistencia de los años 90 era reemplazada por la lectura de que el gobierno de Kirchner abría nuevas posibilidades e implicaba una etapa de "avance popular". Esta interpretación dejaba de lado la lógica reivindicativa de las organizaciones de Trabajadores Desocupados para pensar en una lógica de representación más amplia capaz de llegar a diversos sectores sociales. De esta forma, el desplazamiento de la frontera constituida en los años previos entre las organizaciones y el Gobierno como representante del neoliberalismo, ubicó a este último como la otredad, quedando el primero en una zona que permitiría luego la articulación.

Esos puentes discursivos que construyeron las organizaciones los estructuraron en torno a esas experiencias y trayectorias que conforman un marco de posibilidades compartido para las reconfiguraciones identitarias. Ese espacio convergente expresa la valorización de "lo nacional", de la transformación "gradual" de las sociedades, de una visión latinoamericanista de la política, de la identificación del sujeto Pueblo como el eje vertebrador de la transformación social, de la concepción del peronismo que, más allá de las discusiones no menores por cierto, marca un sustrato sedimentado común en las organizaciones de diversas tradiciones.

Ahora bien, estas reconfiguraciones se dieron de diversa manera. El primer puente se construyó, en el caso de Libres del Sur, a partir de la interpretación de que el gobierno que asomaba en 2003 posibilitaba el "avance popular". Lo que motorizará entonces la inserción será la expectativa que la organización pondrá en dinamizar lo que entendían era un gobierno "permeable" a las demandas populares. No obstante, esa visión se estructurará sobre una identidad sedimentada que visualizaba históricamente las limitaciones que implicaba que el proceso de transformación fuera conducido por el Peronismo y su reverso negativo, el "pejotismo". 
El segundo puente discursivo se estructuró en la reconstrucción de la tradición plebeya del peronismo que realizó el movimiento Evita. En este caso los elementos sedimentados "latentes" ligados a una interpretación contestataria del peronismo durante los años 90 rápidamente se reactivaron en el nuevo contexto, constituyendo una articulación o fusión con el espacio kirchnerista que acompañó todo el proceso.

Por último, la reconfiguración identitaria de mayor amplitud fue la del Movimiento de Unidad Popular puesto que los puntos nodales con los cuales habían estructurado su identidad anarquista en sus comienzos se desplazaron y quedaron subsumidos a los elementos de la tradición "nacional popular". Con la idea de conversión dimos cuenta del cambio en el discurso de la organización a partir de su inserción. El puente que construye el MUP entonces, con el espacio kirchnerista, tuvo la característica principal de constituirse en una identificación con la identidad que entendían tenía el sujeto al cual aspiraban representar. De esa forma, la mutación y conversión del anarquismo al peronismo se operó desde una idealización e identificación con el segundo. La inserción en el nuevo espacio político implicará una reorientación del discurso y los elementos que estructuraban la identidad anarquista serán desplazados por los de la identidad "nacional y popular". En ese marco, la idea de proyecto nacional, Estado interventor, Pueblo, antiimperialismo, serán los elementos que reconfigurarán esta identidad en el proceso de "peronización".

Al comienzo del artículo nos habíamos preguntado por la posibilidad de construir un concepto de identidad capaz de captar tanto lo sedimentado como lo dinámico de una identidad. De esa forma, los puentes discursivos constituyen un concepto operacional que permite analizar las dos dimensiones de la dinámica identitaria.

Ahora bien, para analizar ese proceso dinámico es necesario retomar la distinción que Laclau (2005: 97) realiza entre identidad y acto de identificación. En este sentido, la identificación constru- 
ye la identidad puesto que la entendemos en tanto articulación de significados para dar sentido a una situación. Esto supone concebir un espacio analítico en la articulación de significados que construyen a partir de la apelación a elementos del campo "nacional y popular" y cómo cada una de las organizaciones articulará los mismos en sus cadenas de significación. De este modo, las identidades son el producto de determinadas articulaciones subjetivas parcialmente estables que incorporan aspectos identitarios, dimensiones de reconocimiento, proyecto y acción (Retamozo, 2008: 32).

Estas reconfiguraciones identitarias son articulaciones de elementos significantes que en distintas coyunturas tendrán distintas posiciones, pero operan dentro de lo que podríamos denominar como una matriz ideológica que son las doctrinas previas que generan una determinada forma de identificación política, es decir, un mínimo denominador común de la matriz ideológica a la que se ven obligadas a legitimar en tanto que base de validez que hace posible su propio discurso (Perelli, 1986: 38).

En ese marco, las identidades reconstruyeron sus cadenas de significación articulando, diferencialmente, los elementos simbólicos y produciendo nuevos sentidos. El exterior constitutivo (Muñoz, 2006: 124) viene a jugar un rol central en la explicación, puesto que es necesario imponer una frontera que permita mostrar qué está dentro y qué no. En los casos analizados se puede observar cómo se corre la frontera del exterior constitutivo que asociaba al Gobierno con Neoliberalismo, para dejar a este último significante dentro de otro espacio político, permitiendo la articulación de estas identidades con el Gobierno.

\section{Conclusiones}

La mirada propuesta apunta a diferenciarse de diversos estudios que realizan un análisis más coyuntural de la acción política de las organizaciones, dejando de lado los procesos de constitución 
de identidades. Lo que aquí se propone en contraposición es la reflexión en torno a la posibilidad de observar a partir de un entramado teórico la heterogeneidad de distintas trayectorias que confluyen a partir de operaciones discursivas diferentes.

La profundización en el recorrido histórico implica una búsqueda que trascienda las identificaciones coyunturales de las organizaciones en el presente. Aquí es central recuperar la concepción experiencial ${ }^{14}$ que propone Grimson (2004: 181), que destaca que una identidad se conforma a partir de las experiencias históricas "marcantes" que son constitutivas de modos de imaginación, cognición y acción. Las experiencias históricas que las organizaciones comparten sedimentan articulando y conformando sus identidades como resultado de un proceso histórico social.

La recuperación de las trayectorias políticas de las organizaciones y las experiencias previas posibilitó reconstruir las tradiciones, las instancias de decisión, reactivación y sedimentación para comprender las diversas articulaciones de los movimientos en la disputa por la hegemonía. Es central recuperar los sucesivos aprendizajes y posicionamientos, es decir, las experiencias políticas de los movimientos para la explicación del proceso abierto en 2003.

Cada nueva instancia decisoria por la que atravesaron las organizaciones implicó un proceso en donde estas identidades reconfiguraron sus cadenas de significación. Estos desplazamientos de los elementos constitutivos de las identidades se dieron en lo que denominamos coyunturas, que se caracterizan por ser períodos en donde las organizaciones evalúan las posibilidades de

14 Esta noción se basa en una crítica a las visiones esencialistas y constructivistas de las identidades. Si la concepción esencialista afirmaba que las identidades tenían rasgos objetivos en común, la concepción constructivista tendió a afirmar que las mismas son básicamente "imaginadas". La crítica del autor al constructivismo se centra en que explica que la identidad es una construcción, pero sin embargo, no puede comprender por qué esas construcciones fueron exitosas. 
acción y deciden sus posicionamientos. Es en esas redefiniciones donde se vuelve a reconstruir la identidad a partir de la relectura del pasado y las expectativas que esa coyuntura despierta en las agrupaciones. En esos momentos decisorios las tradiciones y las identidades sedimentadas jugaron un rol central puesto que son estas las que les permitieron a las organizaciones significar las situaciones y escoger en consecuencia ciertas alternativas de acción a través de la decisión.

En ese sentido, el proceso de articulación y reconfiguración de las identidades no puede pensarse por fuera de las experiencias históricas ni tampoco rehuyendo del espacio de libertad-creación que los actos de identificación generan en las identidades. Ese espacio de creación debe analizarse en su confluencia con lo sedimentado en tradiciones y matrices que estructuran un espacio de posibilidades de reactivación. En este aspecto el análisis de la dinámica política a partir del concepto de identidad desarrollado permite reconocer y explicar las acciones.

El análisis de la dimensión política de las identidades creemos constituye un aporte al campo de estudios sobre organizaciones populares y movimientos sociales en la etapa posterior a 2003. En este aspecto, queda abierta la agenda de investigaciones para futuros desarrollos tanto en relación a la dinámica de las organizaciones en etapas que este trabajo no aborda, como para la utilización de este andamiaje teórico como marco para pensar los procesos constituyentes y de reconfiguración de las identidades políticas contemporáneas.

\section{Bibliografía}

ABOY CARLÉS, Gerardo (2001): Las dos fronteras de la democracia argentina. La reformulación de las identidades políticas de Alfonsin a Menem, Rosario, Homo Sapiens.

ARFUCH, Leonor (2002): "Problemáticas de la identidad", en Arfuch, (Comp.) Identidades, sujetos y subjetividades, Buenos Aires, Prometeo. 
ARMELINO, Martín (2007): "Acción colectiva e historia. Notas para el estudio de la acción sindical de ATE (1976-2005)". Cuartas Jornadas de Jóvenes Investigadores, organizadas por el Instituto de Investigaciones Gino Germani, Facultad de Ciencias Sociales (UBA), 19 al 21 de septiembre de 2007, Buenos Aires.

BARROS, Sebastián (2006): “Espectralidad e inestabilidad institucional. Acerca de la ruptura populista", en Estudios Sociales, Santa $\mathrm{Fe}$, año XVI, $\mathrm{n}^{\mathrm{O}} 30$, primer semestre.

BAUMAN, Zigmunt (2002): Modernidad líquida, Buenos Aires, FCE.

BECK, Ulrich (2002): Libertad o capitalismo, Barcelona, Paidós.

BECK, Ulrich (2004): Poder y contrapoder en la era global. La nueva economía política mundial, Barcelona, Paidós.

BIGLIERI, Paula y PERELLÓ, Gloria (2007): En el nombre del Pueblo. La emergencia del populismo kirchnerista. Buenos Aires, Editorial de la Universidad de San Martín.

BRUBAKER, Rogers y COOPER, Frederick (2001): “Más allá de identidad", en Apuntes de Investigación del CECYP. Año V, No 7, Buenos Aires, Fundación del Sur, pp. 30-67.

DE LA GARZA, Enrique (1997): “Trabajo y Mundos de Vida” en H. Zemelman (coord.) Subjetividad: Umbrales del Pensamiento Social, Madrid, Anthropos.

DE LA GARZA, Enrique (2001): “La epistemología crítica y el concepto de configuración", en Revista Mexicana de Sociología $\mathrm{N}^{\mathrm{o}} 1 / 2001$, pp. 109-127.

DUBAR, Claude (1991): La socialización, París, Armand Colin.

DUBET, Francois (1989): “De la sociología de la identidad a la sociología del sujeto", en Estudios Sociológicos, vol. VII, No 21, México D.F., pp. 519-545.

GIMÉNEZ, Gilberto (1997): “Materiales para una teoría de las identidades sociales". En: Frontera Norte $\mathrm{N}^{\mathrm{o}}$ 18, julio-diciembre, México, El Colegio de la Frontera Norte, pp. 9-28.

GIMÉNEZ, Gilberto (2004): “Cultura e identidades", en Revista Mexicana de Sociología, México, Año 66, Nº especial.

GRIMSON, Alejandro (2004): “La experiencia argentina y sus fantasmas", en Grimson, Alejandro (comp.) La cultura en las crisis latinoamericanas, Buenos Aires, CLACSO, pp. 177-193. 
GURRERA, María Silvina (2005): “La redefinición del conflicto social. La conformación de la Central de Trabajadores Argentinos", en Delamata Gabriela, Ciudadanía y Territorio. Las relaciones politicas de las nuevas identidades sociales, Buenos Aires, Espacio.

JENKINS, Craig (1994): "La teoría de la movilización de recursos y el estudio de los movimientos sociales". En: Zona Abierta, $\mathrm{N}^{\circ}$ 69, Madrid, pp. 5-48.

KLACHKO, Paula (2008): “Reflexiones generales sobre el movimiento de trabajadores desocupados", en las Jornadas Internacionales de Problemas Latinoamericanos, Universidad Nacional de Mar del Plata.

LACLAU, Ernesto (1990): Nuevas Reflexiones sobre la revolución de nuestro tiempo, Buenos Aires, Nueva Visión.

LACLAU, Ernesto (2005): La razón populista, Buenos Aires, FCE.

LACLAU, Ernesto (2006): "Por qué construir un pueblo es la tarea principal de la política radical", en Cuadernos del CENDES, mayoagosto año/vol. 23, núm., 062, políticas públicas, pp. 1-36. http://www.cendes-ucv.edu.ve/pdfs/revista62/cap1.pdf

MC ADAM, Doug, MC CARTY, John, y ZALD, Mayer (1999): “Oportunidades, estructuras de movilización y procesos enmarcadores: hacia una perspectiva sintética y comparada de los movimientos sociales", en Movimientos sociales: perspectivas comparadas, España, Istmo.

MELUCCI, Alberto (1994): “Asumir un compromiso: identidad y movilización en los movimientos sociales", en Zona-Abierta $N^{o}$ 69, Madrid.

MERKLEN, Denis (2005): Pobres ciudadanos: las clases populares en la era democrática 1983-2003, Buenos Aires, Gorla.

MUNCK, Gerardo (1995): “Algunos problemas conceptuales en el estudio de los movimientos sociales", en Revista Mexicana de Sociología $\mathrm{N}^{\circ}$ 3, pp. 17-39.

MUÑOZ, María Antonia (2006): “Laclau y Rancière: algunas coordenadas para la lectura de lo político", Andamios. Revista de Investigación social. vol. 2, Nº 4, México, pp. 119-144.

OFFE, Claus (1992): Partidos políticos y nuevos movimientos sociales, Madrid, Sistema. 
PERELLI, Carina (1986): Matrices ideológicas y discurso politico: el problema de la hegemonía. Montevideo, Documentos de Trabajo, n. 125, Montevideo, CIESU.

PÉREZ LEDESMA, Manuel (1994): “Cuando lleguen los días de cólera (Movimientos sociales, teoría e historia)", en Zona-Abierta No 69, Madrid.

PIZZORNO, Alessandro (1994): “Identidad e Interés", en Zona Abierta, 69, Madrid, pp. 136-143.

RETAMOZO, Martín (2006): El movimiento de trabajadores desocupados en Argentina. Subjetividad y acción en la disputa por el orden social. Mimeo. Tesis de Doctorado, México, FLACSO.

RETAMOZO, Martín (2008): “Los sentidos del (sin) trabajo. Subjetividad, demanda y acción colectiva de trabajadores desocupados en Argentina", en Sociohistórica. Cuadernos del CISH, La Plata, EDULP, pp. 55-89.

RETAMOZO, Martín (2009a): Orden social, subjetividad y acción colectiva. Notas para el estudio de los movimientos sociales, en Athenea Digital: revista de pensamiento e investigación social, Nº 16, 2009, pp. 95-123.

RETAMOZO, Martín (2009b): “Las Demandas Sociales y el Estudio de los Movimientos Sociales", en Cinta de Moebio: Revista Electrónica de Epistemología de Ciencias Sociales, Chile, No 35.

SCHUSTER, Federico (2005): “Las protestas sociales y el estudio de la acción colectiva", en Schuster y otros (comps.) Tomar la palabra. Estudios sobre protesta social y acción colectiva en la Argentina contemporánea, Buenos Aires, Prometeo.

SIDICARO, Ricardo (2003): “Consideraciones sociológicas sobre la Argentina en la Segunda Modernidad", en Estudios sociales, $N$. 24 , primer semestre.

SVAMPA, Maristella (2000): "Identidades astilladas. De la patria metalúrgica al heavy metal", en Svampa, M. (ed.). Desde abajo. Las transformaciones de las identidades sociales, Buenos Aires, Biblos-UNGS, pp. 121-154.

SVAMPA, Maristella (2004): “Cinco Tesis sobre la nueva matriz popular", en revista Laboratorio/on line. Revista de Estudios Sobre Cambio Social, año IV, número 15, primavera.

SVAMPA, Maristella (2005): "La Sociedad Excluyente. La Argentina bajo el signo del neoliberalismo", Buenos Aires, Taurus. 
TARRÉS María Luisa (1992): “Perspectivas analíticas en la sociología de la acción colectiva", en Estudios Sociológicos, Volumen 10, $\mathrm{N}^{\circ}$ 30, septiembre-diciembre.

TARROW, Sydney (1997): El poder en movimiento, Madrid, Alianza.

TARROW, Sydney (1999): “Estado y oportunidades: la estructuración política de los movimientos sociales" en Mc Adam, Doug; Mc Carty, John, y Zald, Mayer, Movimientos sociales: perspectivas comparadas, España, Istmo.

TOURAINE, Alain (1991): Los Movimientos Sociales, México, Almagesto.

TORRES CARRILLO, Alfonso (2006): “Organizaciones populares, construcción de identidad y acción política", en Revista Latinoamericana de Ciencias Sociales, Vol. $4 \mathrm{~N}^{\circ}$ 2, Colombia, Universidad de Manizales.

ZEMELMAN, Hugo (1995): “La esperanza como conciencia (un alegato contra el bloqueo histórico imperante: ideas sobre sujetos y lenguaje)", en Zemelman (coord.). Determinismos y Alternativas en las Ciencias Sociales de América Latina, México, CRIM-UNAM.

ZEMELMAN, Hugo (1997): "Sujetos y subjetividad en la construcción metodológica", en León y Zemelman (coords.), Subjetividad: Umbrales del Pensamiento Social, Barcelona, Anthropos, pp. 21-35. 